\title{
Research on Flow Pattern of Nitrogen Tetroxide Liquid in the Different Bend Radii Pipes
}

\author{
Pengfei Hao, Wenji Si, Hui Zhang, Ping Zhang, Shouti Yue \\ Department of Technique, Taiyuan Satellite Launch Centre, Taiyuan, Shanxi, China
}

\begin{abstract}
N}_{2} \mathrm{O}_{4}$ is a common rocket fuel propellants, it has the characteristics of low boiling point and a large viscosity, the friction between viscosity fluids and pipeline dramatic leads to a huge sticky heat, therefore, the vaporization phenomenon often occurs in the pipeline, particularly in bending of the viscous heat. For this reason, the research of the different bending radii vaporized fluid conditions for optimizing the piping and precise the filling flow is significant. In this paper, the MIXTURE mixed flow model is used to achieve the numerical simulation the pipelines filling of the three different bending radii, it still have not solved the mass transfer problem between the different phases. Therefore, the custom functions are needed to define the mass transfer problems from the liquid phase to the vapor phase. Though the contrast among the volume phase cloud of six different elbow models, we have the following conclusions: 1) In the entire pipeline transportation, the distribution vaporization rate from the inlet pipe to the outlet pipe follows the distribution of the first increasing and then decreasing, the gas rates of the elbow area is highest; 2)Analyzing the sticky heat for different bend radii, we have the conclusion that the lowest bending vaporization the of the optimal radius is $0.45 \mathrm{~m}$. The above conclusions are drawn in good agreement with the actual law, can effectively guide the engineering practice, have important significance for the future design for the optimization of the fuel pipeline transportation.
\end{abstract}

\section{Introduction}

Nitrogen tetroxide $\left(\mathrm{N}_{2} \mathrm{O}_{4}\right)$ has strong oxidation,its available oxygen can be up to $70 \%$, it can be spontaneous in contact with amines, hydrazine, alcohol and many other fuels. Therefore it often used as bipropellant liquid propellant oxidizer. Currently, in the military aspects, unsymmetrical dimethyl hydrazine (UDMH) and $\mathrm{N}_{2} \mathrm{O}_{4}$ are combined at room temperature to raise the rocket chamber. $\mathrm{N}_{2} \mathrm{O}_{4}$ has huge sticky heat, therefore, the pressure in the pipe will produce viscous heat transport, the boiling point of the substance is 21.2C.Therefore, long-range transport in the pipeline is likely to occur vaporization phenomenon due to the viscous heat, thereby reducing the transportation flow throughout the pipeline, particularly in bending of the viscous heat. The main content of this paper is the optimal bend radius of the filling system, that find out that under any bend radii, the number of the vaporization due to the sticky heat produced by $\mathrm{N}_{2} \mathrm{O}_{4}$ is lowest.

Currently, the fluid researches are focused on lowviscosity fluids. Thus, the practical study does not need to consider the viscous heat problem. The main reason that the vaporization phenomenon occurs in the elbow is that fluid cavitation. In this research, there also exists some cavitation in elbow. However, compared with the vaporization due to the viscous heat, it can be negligible.
The CFD numerical simulation method is adopted to study the optimal radius of the filling systems.

Zhan Han-hui containing [6], under different flow conditions ,the secondary flow (Dean Vortex) of the 90 elbow inside is simulated, the simulation process uses the $k \sim \mathcal{E}$ model with a swirl correction is calculated to obtain the pressure and speed distribution of the different flow regimes in flow direction of the cross-section; Shi Feng [7] etc., do the study $R_{c} / d=1.69$ ( $R_{c}$ is the average radius of the bend, $d$ is the Hydrodynamic diameter elbow) turbulent flow in the $90^{\circ}$ bend based on the Standard $k \sim \varepsilon$ turbulence model, it is found when using the model to get the flow field velocity distribution compared with experimental data, in the bend $\theta=45^{\circ}$, both are in good agreement, in other words the calculation error is small, in the bend $\theta>45^{\circ}$, there are larger error. Ding Jue [8] etc., use 3 turbulence model(the Standard $k \sim \varepsilon$ turbulence model, $\mathrm{RNG}$ $k \sim \varepsilon$ turbulence model, Realizable $k \sim \varepsilon$ turbulence model),simulation a square cross-section threedimensional turbulent flow in the $90^{\circ}$ large curvature of the curved pipe, RNG $k \sim \varepsilon$ turbulence model and twowall model are more applicable in the numerical simulation of turbulent considered strong curvature of the 
bend. The above findings are not considered viscous heat production, therefore, to study the subject has some innovative.

\section{Control equations}

\subsection{Multiphase flow mixing equation}

This paper uses a mixed flow model for two-phase flow simulation,the mixed is mixed liquid and gas phase, it variable density and ignore the relative movement between the liquid and gas,flow field calculation just needs a control equation that is the mixed phase equations, gas phase volume distribution can be obtained by gas phase mass transport equations.

Mixed phase continuity equation:

$$
\begin{gathered}
\frac{\partial}{\partial t}\left(\rho_{m}\right)+\nabla \cdot\left(\rho \overline{V_{m}}\right)=0 \\
\rho_{m}=\rho_{v} \alpha+\rho_{l}(1-\alpha)
\end{gathered}
$$

In the equation: $\rho_{m}$ is the mixed phase density; $V_{m}$ is the mixed phase velocity.

Mixed phase momentum equation:

$$
\begin{aligned}
& \frac{\partial}{\partial t}\left(\rho \overrightarrow{V_{m}}\right)+\nabla \cdot\left(\rho \overrightarrow{V_{m}} \overrightarrow{V_{m}}\right)= \\
& \vec{F}-\nabla p+\nabla \cdot\left[\mu_{m}\left(\nabla \overrightarrow{V_{m}}+\left(\nabla \overrightarrow{V_{m}}\right)^{T}\right)\right]
\end{aligned}
$$

Gas phase transport equation:

$$
\frac{\partial}{\partial t}\left(\rho_{m} f\right)+\nabla \cdot\left(\rho_{m} f \overrightarrow{V_{m}}\right)=m_{e}-m_{c}
$$

In the equation: $f$ is the gas phase mass fraction; $m_{e}$ is the vaporization source term ; $m_{c}$ is the Condensation source term.

\subsection{Energy equation}

FLUENT Solving the following energy equation:

$$
\begin{aligned}
& \frac{\partial}{\partial t}(\rho E)+\nabla \cdot(\vec{u}(\rho E+p))= \\
& \nabla \cdot\left(k_{\text {eff }} \nabla T-\sum h_{j} \overrightarrow{J_{j}}+\left(\overline{\overline{\tau_{\text {eff }}}} \cdot \vec{u}\right)\right)+S_{h}
\end{aligned}
$$

In the equation, $k_{\text {eff }}$ is the effective thermal conductivity ; $\quad \vec{J}_{j}$ is the diffusion flux of constituent $j$.The first three to the right of equation, respectively, due to thermal conduction, species diffusion, viscous dissipation arising energy transfer. $S_{h}$ containing chemical reactions release (absorption) heat and any other user-defined volumetric sources.

$$
E=h-\frac{p}{\rho}+\frac{u^{2}}{2}
$$

For incompressible fluid:

$$
h=\sum_{j} Y_{j} h_{j}+\frac{p}{\rho}
$$

$Y_{j}$ is the mass fraction of constituent $j$.

$$
h_{j}=\int_{T_{r e f}}^{T} c_{p, j} d T
$$

$T_{\text {ref }}$ is $298.15 \mathrm{~K}$

Equation contains the viscous dissipation term, which represents the heat generated by the viscous shear in the flow process. When using the segregated solver, the default energy equation does not contain this one in FLUENT (because generally ignored the heat generated by viscous). When Brinkman, Close to or larger than 1, the Viscous fluid heat can not be ignored:

$$
B r=\frac{\mu U_{e}^{2}}{k \Delta t}
$$

$\Delta t$ is the temperature difference calculated within the region.

\subsection{Mass transport model}

The mixture model of multiphase flow to Fluent, still can not solve the problem in a different mass transfer between phases. So, we need to use a custom function (UDF) to define the mass transfer problems from the liquid phase to the steam phase. To do this, on the one hand we define the mass absorption phase in the steam continuity equation, on the other hand we define the equivalence of mass source term in the liquid continuity equation.

\section{Mathematical model}

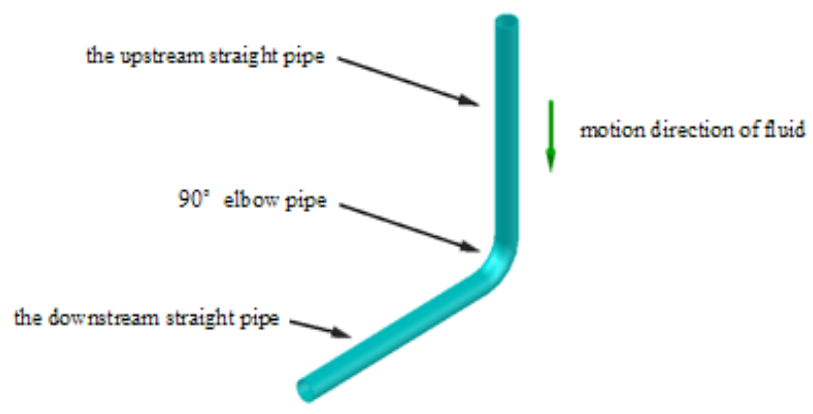

Figure 1. Mathematical model in the simulation region 
Mathematical model consists of Upstream straight pipe, 90 degree elbow segment and downstream straight pipe three parts for the pipeline to transport, in order to ensure the model is comparable, upstream and downstream straight pipe sections were maintained at $1 \mathrm{~m}, 90$ degree bend segments selected six different radius to simulate, the diameter of the entire pipe is $0.1 \mathrm{~m}$, the radius 90 degree bend segments selected are $0.15 \mathrm{~m}, 0.25 \mathrm{~m}, 0.35 \mathrm{~m}$, $0.45 \mathrm{~m}, 0.55 \mathrm{~m}$ and $0.65 \mathrm{~m}$.

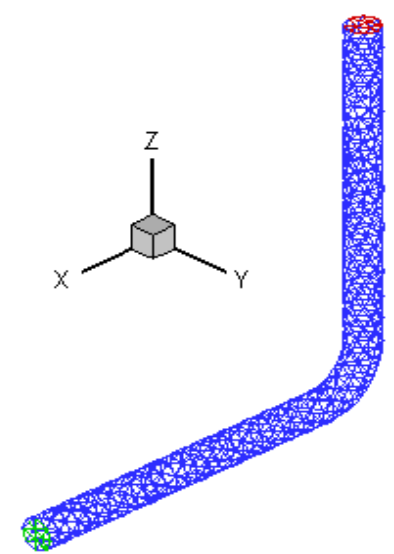

Figure 2. Computational domain and the mesh

To do the three-dimensional solid modeling using Gambit. The model handles the viscous heat problem, that is larger friction between the wall and the fluid, so have the need to encrypt boundary of the inner wall to the pipeline, the first layer thickness of the boundary is $0.001 \mathrm{~m}$, the thickness growth rate between the two layers is 1.2 , in total four layers, the thickness of the boundary layer is $0.005368 \mathrm{~m}$.In order to make the numerical calculation facilitate,the entire mesh of the calculation model take the tetrahedral grid structure, the number of the mesh is $8,532,450,000$.

The main simulation object in this paper is $\mathrm{N}_{2} \mathrm{O}_{4}$, so, we need o modify material properties in the FLUENT,

\begin{tabular}{|c|c|}
\hline Physical parameters & Value \\
\hline $\begin{array}{c}\text { Liquid Density }\left(21.1^{\circ} \mathrm{C},\right. \\
101.325 \mathrm{kPa})\end{array}$ & $1443 \mathrm{~kg} / \mathrm{m} 3$ \\
\hline $\begin{array}{c}\text { Gas density }\left(21.1^{\circ} \mathrm{C},\right. \\
101.325 \mathrm{kPa})\end{array}$ & $3.40 \mathrm{~kg} / \mathrm{m} 3$ \\
\hline $\begin{array}{c}\text { Specific heat capacity (gas, } \\
101.325 \mathrm{kPa})\end{array}$ & $\mathrm{Cp}=831.5 \mathrm{~kJ} /(\mathrm{kg} \cdot \mathrm{K})$ \\
\hline Viscosity $\left(101.325 \mathrm{kPa}, 25^{\circ} \mathrm{C}\right)$ & $0.0132 \mathrm{mPa} \cdot \mathrm{S}$ \\
\hline Viscosity $\left(\right.$ liquid, $\left.20^{\circ} \mathrm{C}\right)$ & $0.42 \mathrm{mPa} \cdot \mathrm{s}$ \\
\hline $\begin{array}{c}\text { Thermal conductivity } \\
\left(101.325 \mathrm{kPa}, 50^{\circ} \mathrm{C}\right)\end{array}$ & $0.16747 \mathrm{~W} /(\mathrm{m} \cdot \mathrm{K})$ \\
\hline
\end{tabular}

This calculation model take the two-phase Mixture Model to simulate, the model is applicable to the calculation of two-phase flow due to vaporization phenomena, for cavitation, the interaction command in the model can solve, there need to fill the corresponding saturated vapor pressure and the surface tension coefficient of the fluid.For the calculation of two-phase flow, the problem of quality transmission still can not be solved, need to customize UDF programming to resolve.
The key program of $\mathrm{C}$ language:

if $\left(\mathrm{C}_{-} \mathrm{T}(\right.$ cell, mix_th $\left.)>=\mathrm{T} \_\mathrm{SAT}\right)$

$\left\{\begin{array}{l}\left.m_{-} d o t_{-} v=0.1 \cdot C_{-} V O F(\text { cell, pri_th }) \cdot C_{-} \text {R(cell, pri_th }\right) \\ \cdot \operatorname{fabs}\left(\mathrm{C}_{-} \mathrm{T}(\text { cell, mix_th })-\mathrm{T}_{-} \mathrm{SAT}\right) / \mathrm{T} \_\mathrm{SAT} ; d S[\text { eqn }]=0 . ;\end{array}\right\}$

else

$\left\{\begin{array}{l}m_{-} d o t_{-} v=-0.1 \cdot C_{-} V O F(\text { cell, sec_th }) \cdot C_{-} \mathrm{R}(\text { cell, sec_th }) \\ \cdot \mathrm{fabs}\left(\mathrm{T} \text { SAT }-\mathrm{C} \_\mathrm{T}(\text { cell, mix_th })\right) / \mathrm{T} \_\mathrm{SAT} ;\end{array}\right.$ $\left.\begin{array}{l}d S[\text { eqn }]=-0.1 \cdot C_{-} R(\text { cell, sec_th }) \\ \cdot \operatorname{fabs}\left(\mathrm{C}_{-} \mathrm{T}(\text { cell, sec_th })-\mathrm{T}_{-} \mathrm{SAT}\right) / \mathrm{T}_{-} \mathrm{SAT} ;\end{array}\right\}$

In which $T_{-} S A T$ is the Vaporization temperature, through the above $C$ language programming, to realize the equal mass exchange of gas-liquid two-phase material ,otherwise, will arise the conserved problem to the continuity equation, cause unnecessary problems to the Calculation.

The import border is set to the entrance of the speed in the calculation, the flow rate of the fluid in the pipe is $5 \mathrm{~m} / \mathrm{s}$, we use outflow free flow boundary conditions in outlet .Take the no-slip boundary conditions to the wall, do discrete based on the finite volume method to equations,take the second-order upwind to convection term, use second-order central difference scheme diffusion, take Simplec algorithms to pressure-velocity coupling, unsteady discrete format is a first-order implicit scheme, the time step is $0.001 \mathrm{~s}$.

\section{Numerical calculation}

In order to study the sticky heat vaporization phenomenon of the pipelines better under different conditions bend radius, we have six different flow field bend radius numerical simulation calculations.

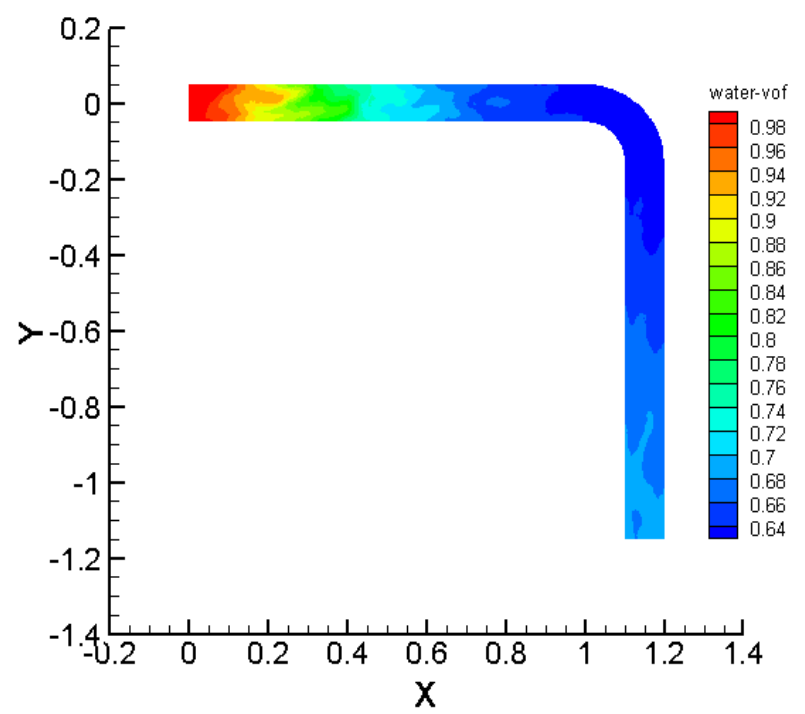

Figure 3-a. Bend radius $0.15 \mathrm{~m}$ 


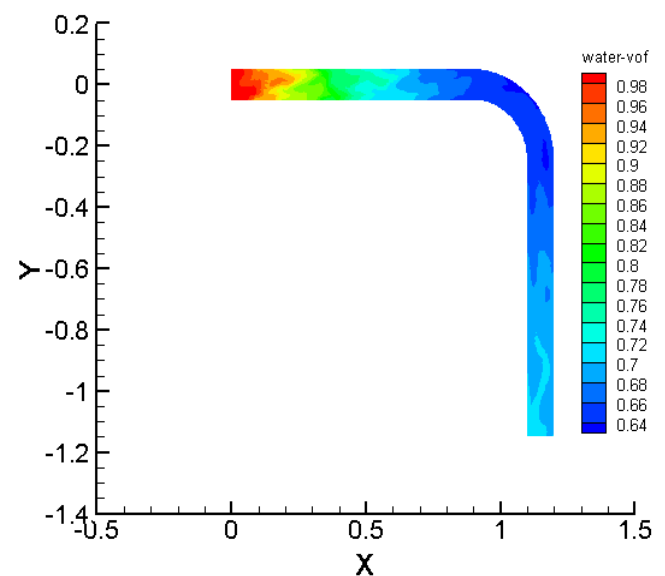

Figure 3-b. Bend radius $0.25 \mathrm{~m}$

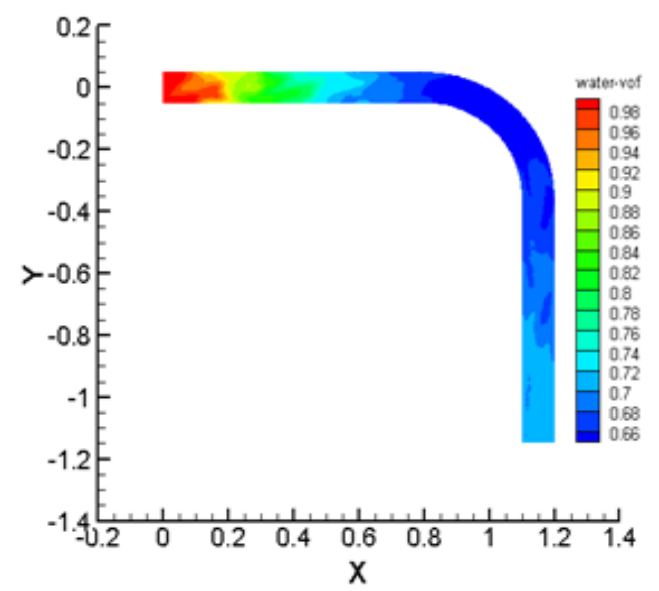

Figure 3-c. Bend radius $0.35 \mathrm{~m}$

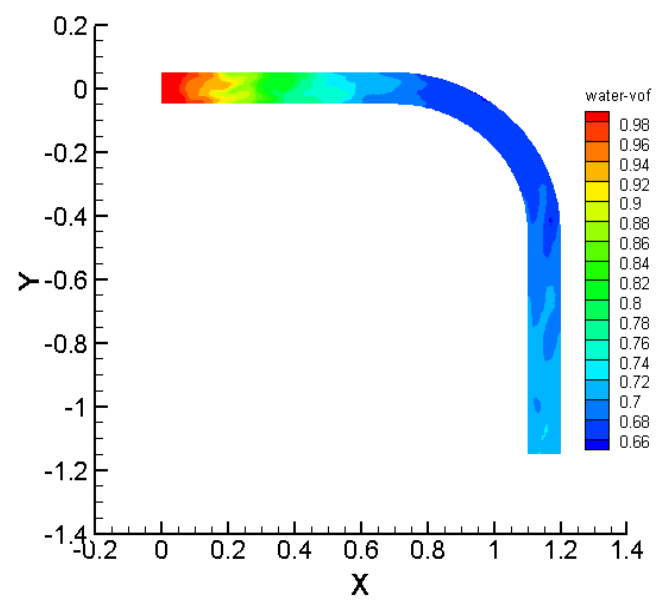

Figure 3-d. Bend radius $0.45 \mathrm{~m}$

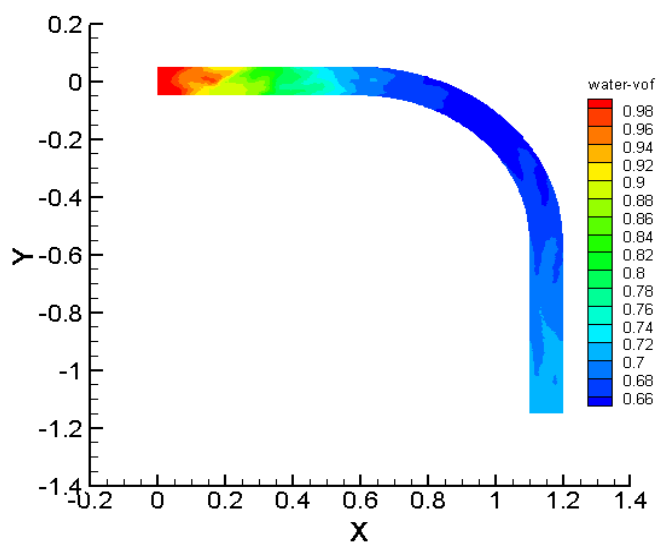

Figure 3-e. Bend radius $0.55 \mathrm{~m}$

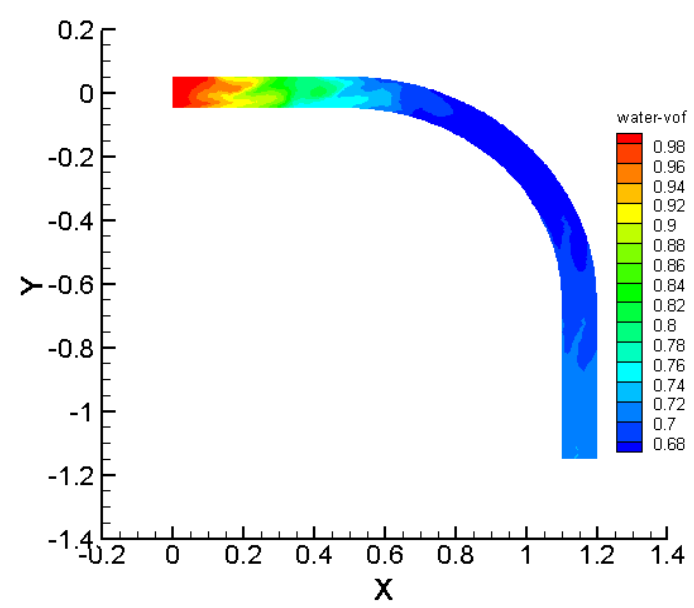

Figure 3-f. Bend radius $0.65 \mathrm{~m}$

From Fig. 3, the vaporization in elbow is more serious, the distribution vaporization rate from the inlet pipe to the outlet pipe follows the distribution of the first increasing and then decreasing. The main reason is: $\mathrm{N} 2 \mathrm{O} 4$ fluid viscosity is greater, the intense friction between the high-speed flow wall, results in a viscous heat, achieves the vaporization temperature of the fluid, the fluid will occur vaporization in section, aeration pipelines to some extent reduce the total transport fuel, this brings some inconvenience to the rocket fuel filling cavities. The fluid in the pipe suffers the action of centrifugal force, The temperature produced by great force generating of the inner wall of the outer fluid pipe is higher, so as the serious vaporization.

As can be seen from Fig. 4, of the distribution of the pipe viscous heat of vaporization first decreases and then increases the with vaporization increasing of the bending radius, there exists optimal pipe radius of the minimum amount of the vaporization. The vaporization distribution of the elbow is related to the length of the elbow and the total length of the transmission pipelines, the longer distance of elbow the more serious of the turbulent fluid, however, the longer the elbow the total length of the entire pipe will be reduced,friction distance is shorter between the fluid and pipeline,viscous heat generation is reduced,in both factors simultaneously, there must exist a more reasonable bend radius.From the figure it can be drawn when the bending radius is $0.45 \mathrm{~m}$,viscous heat is the least.

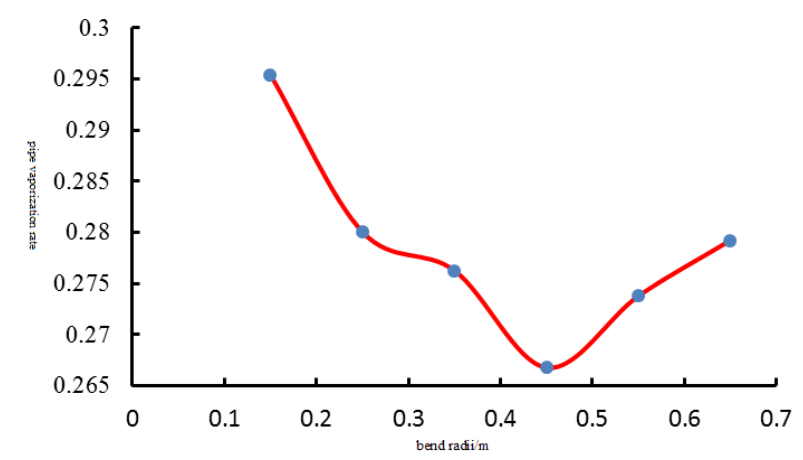

Figure 4. Vaporization distribution in the different bend radii pipeline 


\section{Conclusions}

In this paper, the MIXTURE mixed multiphase flow model and modified $R N G \quad k \sim \varepsilon$ turbulence model are used to simulate the three-dimensional model of the fuel filling pipe elbows. The mass transport problem can not be solved by this model, thus we have to write $U D F$ mass transport equations. Six different transport pipeline bend radius of the fluid is simulated, we obtain the distribution of the two-phase flow at different radii bends and the same time, The pipeline and the pipeline serious vaporization zone vaporization rates are compared and analyzed, we have the following conclusions:

1) In the entire pipeline transportation, the distribution vaporization rate from the inlet pipe to the outlet pipe follows the distribution of the first increasing and then decreasing, the gas rates of the elbow area is highest;

2) Analyzing the sticky heat for different bend radii, we have the conclusion that the lowest bending vaporization the of the optimal radius is $0.45 \mathrm{~m}$.

The above conclusions are drawn in good agreement with the actual law, can effectively guide the engineering practice, have important significance for the future design for the optimization of the fuel pipeline transportation.

\section{References}

1. L. J. Xie, Si-yu, J. G. Yu, PIV Analysis of Inner Flow Field in $90^{\circ}$ Bending Duct of Circular-Section with Fore-End Valve, Journal of Shanghai Jiaotong University, 45(9), 1395-1399, (2011)

2. J. Ding, P. F. Weng, Numerical simulation of theoretical models \& flow characteristics in $90^{\circ}$ bending duct, Chinese Journal of Computational Mechanics, 21(3), 314-321, (2004)
3. Y. Kliafas, T. M. Holt, LDV measurements of a turbulent air-solid two-phase flow in a 90 degree bend, Expriments in Fluids, 5(2), 73-85, (1987)

4. K. P. Taylor, J. H. Whitelaw, M. Yiannes, Curved ducts with strong secondary motion: velocity measurements of developing laminar and turbulent flow, Journal of Fluids Engineering, 104, 350-359, (1982)

5. Y. J. Zhao, Y. Y. He, Hydraulic characteristics of Ztype pipe combination with two similar rectangular bends, Hydraulic Engineering, 37(7), 778-783, (2006)

6. H. H. Zhan, H. Zhu, J. D. Chen, Numerical Simulation of secondary flow (dean vortices) in $90^{\circ}$ curved tube, Boiler Technology, 41(4), 1-5, (2010)

7. F. Shi, Z. Xu, C. F. Ma, Turbulent Flow in Curved Duct with Large Curvature Numerical Computation and Measurement, Acta Aerodynamica Sinica, 8(4), 423-429, (1990)

8. J. Ding, P. F. Weng, Numerical Study on Three Dimensional Turbulent Separated Flow in Rightangled Curved Duct by Three Turbulent Models, Chinese Journal of Computation Physics, 20(5), 386390, (2003)

9. ANSYS Inc. FLUENT User's Guide, Lebanon: Fluent Inc, (2003)

10. L. L. Wang, J. Lu, H. Zhu, Three-dimensional large eddy simulation of water wave using the dynamic coherent structures eddy model, Huazhong University of Science and Technology(Natural Science Edition), 39(1), 106-109, (2011)

11. R. L. Bass., E. B. Bowles, R. W. Trudell, et al. Modeling criteria for scaled LNG sloshing experiments, Transactions of the ASME, 107, 272280, (1985)

12. O. M. Faltinsen, A numberical nonlinear method of sloshing in tanks with two-dimensional flow, Journal of Ship Research, 22(3), 193-202, (1978) 